\title{
Fog Based Sensing and Transmission Mechanisms of Vital Bio-Signals
}

\author{
Adwitiya Mukhopadhyay, Vaibhav B N
}

\begin{abstract}
Internet of Things (IoT) makes everything interconnected to communicate and perform tasks. Some IoT examples to quote are smart city, smart house, smart bus etc. The main focus of this paper is to make a survey and propose a system that automates bio-signal sensing in healthcare industry. The data which are recorded should be accurate and should reach the destination in time for decision making. This can be achieved by using precision microcontrollers enhanced with IoT and fog computing. Using IoT in healthcare industry makes these processes faster and more accurate. Keeping the existing systems in mind we propose system which is a combination of IoT and fog computing. The proposed system can be divided into 4 layers: data gathering, data transmission, data storage and data retrieval. Usage of proposed system in healthcare industry can improve the efficiency of transmitting vital data to healthcare centers during emergency.
\end{abstract}

Index Terms, IoT, Fog Computing, Biomedical Sensors, e-Health, Bio-signal, Microcontrollers, Arduinos.

\section{INTRODUCTION}

In India, health emergency cases are increasing day by day. To treat this kind of cases, the patient should be shifted or should be taken to the hospitals which have complete facilities to treat them. This process needs a sufficient number of ambulances to be present in that area and also well-trained paramedics to take care, observe the patient and record their vital data on the run. But there might be a situation where a paramedic is not well trained and does not know how to treat the patient in some emergency scenarios. Such scenarios can be handled when there is a doctor in contact and help the paramedic telephonically, or by deploying the system that allows the doctor to see the patient's vital data in real-time and also alert the doctor during the high emergency.

The system mentioned above represent the technology like IoT and fog computing. There are n number of IoT products getting released into the market every day. These products make many process fast, automate and avoids many human mistakes. The product based on IoT in healthcare industry overcomes many disadvantage like delay in data transmission or processing, storage issues and etc.

There are many researches done on healthcare IoT. There are many products in the market such as biomedical devices and wearable devices which record the vital information like BP (Blood pressure), Pulse rate, Spo2 (Oxygen saturation), muscle activity and ECG (Electrocardiogram) of an individual. Such devices record the individual data continuously and have a separate database to store these values. The values stored can be accessed by the individual or by the doctors during the time of treatment

Revised Manuscript Received on November 05, 2019.

Adwitiya Mukhopadhyay: Department of Computer Science, Amrita School of Arts and Sciences, Mysuru, Amrita Vishwa Vidyapeetham, India

Vaibhav B N: Department of Computer Science, Amrita School of Arts and Sciences, Mysuru, Amrita Vishwa Vidyapeetham, India or while taking decisions. Decision making plays a very vital role in the healthcare industry. The system deployed should be efficient enough to sense and send the data to the destination or hospital in time.

This paper presents a survey of different kinds of technologies and devices or sensors that are used to build such systems. It also performs a comparison between them, as well as depicts the challenges while implementing the system and how to overcome them.

The paper is organized as follows section II. An introduction to IoT, section III. An introduction to fog computing, section IV. About biomedical sensors and fog nodes and section V. Proposed system.

\section{IOT}

"Internet of Things is an ecosystem of connectedphysical objects that are accessible through the internet" [1]. In a simple word, anything that is connected to the internet and has a capability to communicate with each other is called iot. The 'thing' can be a mobile phone or any other devices or sensors.

Fig 1 shows the hierarchy of IoT and data flow to the cloud. IoT in healthcare aims at real time tracking and monitoring of patients using smart applications.

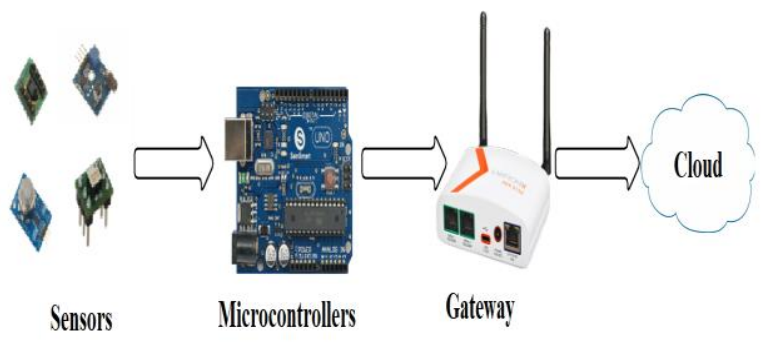

Fig. 1 Basic IoT architecture

Vandana Milind Rohokale et al. [2]mainly focuses on the healthcare facilities provided in the rural areas. The people who are registered to rural healthcare center (RHC) will be given a device and that device alerts the patient and the RHC doctor when certain criteria in their vitals reached. Once the doctor receives the notification he will allots a staff to check the patient's condition.

Dr.A.A.Gurjar et al.[3] Proposed a system that early predicts the heart attack. The proposed system consists of 2 ends one is the transmitter end which consists of the hear sensor attached to the patient body and high and low level will be fixed and the other end is the receiver end where the doctor can monitor patient data and gets alerts when the heart rate reaches high or low level.

Monisha S et al.[4] Proposed a system that early detects the stroke. There is a kind of stroke called wake up stroke which occurs during the sleep. Some set of patients are neglected as the onset time is not clear in this 


\section{Fog Based Sensing and Transmission Mechanisms of Vital Bio-Signals}

kind of stroke. The proposed system consists of the bio-medical sensor to predict the symptom of stroke and alert the patient by alarming before the stroke so that they can take a proper medication to avoid that.

Nirmal Raj M et al.[5] Mainly focuses on the illness called epilepsy and its symptom called seizures. Epilepsy is a kind of brain disorder which makes the patient unconscious or change their behavior. During this time the patient cannot call anyone for a help. To avoid this problem here is a proposed system which records the patient behavior and vitals to alert the doctor or the concerned person. The proposed system consists of sensors like accelerometer sensor to sense the patients fall and the body shake, temperature sensor to record the vital of patient.

Patients who are suffering from some nervous problem lacks the sensation of urinary fullness which affects the patient condition. To avoid that R. Harikrishnan et al.[6] Proposed a system that detects the urinary level of the patient and alerts the doctor or the concerned person. The proposed system consists of ultrasonic sensor attached to the microcontroller to sense and record the level. Once the sensed data reach certain level the system alerts the doctor or the concerned person.

B. N. Karthik et al.[7] Proposed a system which mainly aims on the metaphysics monitoring of the patients and to recommend a workout plan for the patients. Paper also explores some comparative study on different technologies and the limitations. The proposed system consists of heart sensor and temperature sensor to record the vitals of the patients and that data will be send to the thinks speak cloud via esp8266 microcontroller. This recorded data can be accessed by the doctor and suggest a proper workout plan and treatment.

Kuo-hui yeh et al.[8] proposed a secure system which consists of 3 layers of communication i.e. Biomedical sensors, LPU (local processing unit) and BSN (Body Sensor Network). For the communication to happen between biomedical sensor and LPU they should register with the $\mathrm{BSN}$ and after the registration a unique authentication Id will be generated. With the help of that authentic Id communication takes place between them securely.

A. Mukhopadhyay et al. [9] proposed a system that makes use of a ECG monitoring device which was developed by R. Krishnan et al. [10] to record data using an android app. The acquired data will be filtered, analyzed and stored in firebase. The ECG graph is plotted in real-time at the doctor's end.

J. Sathish Kumar et al.[11] mainly focuses on the data security and privacy. Also depicts security concerns like unauthorized access to data, threat by the internet, denial of service, virus or malware affect, replacement of operators etc. Also divides privacy into 3 aspects based on IoT, Device and Communication.

Charalampos Doukas et al. [12] proposed a system in which wearable devices and mobile sensors collects the data of the patients and traverse it to the cloud. The communication happens only between the cloud and component. The data security is ensured by encrypting the data and has also used authentication mechanism to secure the communication.

Moeen Hassanalieragh et al. [13] proposed a simple system of monitoring and managing the patients vital information. This process has been divided into 3 parts in the paper as data acquisition, data transmission, and cloud processing. In data acquisition the vital info of the patients is recorded from different biomedical sensors. In data transmission the data recorded will be transmitted and stored into the cloud. In cloud the data will be processed and will be visualized.

Other example of IoT in healthcare are, cancer treatment using a device called CYCORE which is Bluetooth enabled device which records blood pressure of the patient and also has the symptom tracking app which tracks the vital data in weekly basis.

Continuous glucose level monitoring system is a device which keeps tracks of the blood glucose level of the diabetic patients by recording it continuously.

An IoT product for asthma patient called propeller health is a connected inhaler which connects to the app and help the asthma patients to understand what is causing their symptom.

Connected contact lenses is a product or idea announced by google science in the year 2014, the product could measure the eye glucose level and alert the individual before he/she is prone to diabetes.

Depression monitoring system is apple wearable product which connects to the app to monitor and assess patients with major depressive disorder[14].

Fig 2 represents the IoT in healthcare industry.

Benefits of using IoT in Healthcare industry [15]:

- Low cost.

- By using IoT doctor gets all the required data of the patient that will lead to quality diagnosis and also patients will get more engaged in the treatment.

- Automates many tasks and minimizes the human intervention.

- As the human intervention is minimized there will be less errors or wastages.

- Improved outcome of the treatment.

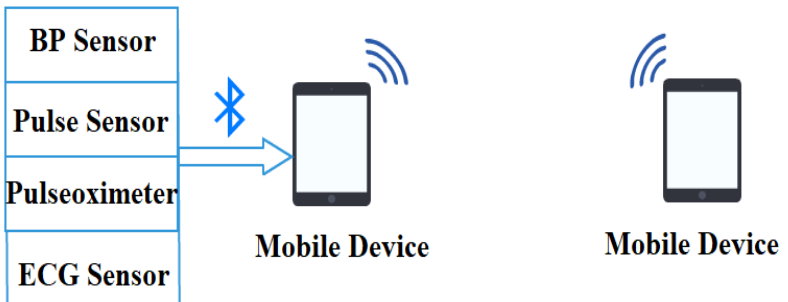

Fig 2. IoT in healthcare

\section{FOG COMPUTING}

"Fog computing, also known as fog networking or fogging, is a decentralized computing infrastructure in which data, compute, storage and applications are distributed in the most logical, efficient place between the data source and the cloud" [16]. In simple words the fog computing is a hierarchy of the devices which has the capability to process and store the data locally and also has the cloud as the backbone. 


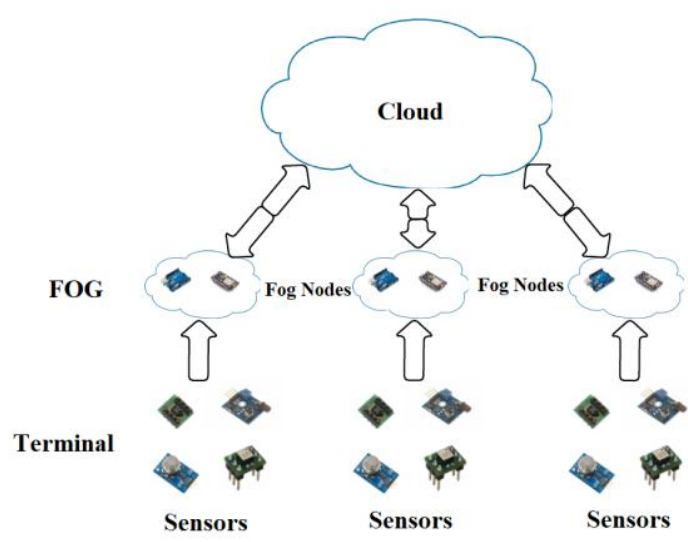

Fig 3. Basic architecture of fog computing

Tilak Patel et al.[17] Mainly conducts the survey on the state of the fog computing, its challenges, its applications and also how it solves the disadvantages of cloud computing. The paper also states some of the need of fog computing in IoT.

Jabril Abdelaziz et al.[18] Mainly focuses on the creation of fog model. The paper depicts how middleware, operational layer and ecosystem work in fog computing. The paper also explains the architecture by giving the example use case of flood warning system.

Md. Redowan Mahmud et al.[19] Mainly focuses on creating a latency- aware application police which specifies the amount of data to be sent or received at the particular unit of time for all application. This ensure the QOS (Quality of Service) of an application.

Gilsoo Lee et al. [20] proposed a secure fog network and also identified the latencies. Here the fog network is divided in to 3 layers: sensing layer, fog layer and cloud storage. The sensing layer consists of $n$ number of sensor which offloads the sensed data to the fog layer. In fog layer there are $n$ number of fog nodes. These fog nodes should accept the data coming from sensing layer and then store it, process it and also traverse it to the next fog node. Here the cloud part is located remotely so the fog node should communicate each other to manage the data.

Subhadeep Sarkar et al. [21] proposed a model of fog network and performs comparative based on latency and power consumption with respect to Cloud computing.

Mung Chiang et al. [22] depicts the various kinds of challenges in fog computing from security, network and resource aspects. It also represents the Challenges of cloud computing and how that is solved using fog computing.

Y.Navaneethkrishnan et al. [23] depicts some of the disadvantages of cloud computing and solves that disadvantages using an working model of fog computing. Here the working model consists of 2 scenarios where every sensed data is directly stored in cloud and the other scenario is using a node as fog. Here the comparison is done between these 2 scenarios and results are shown.

T. N. Gia et al. [24] suggests a system that enhance the health monitoring system. This also uses fog computing concept. The main aim of this system is to provide a smart gateway that includes the services like data mining and distributed data storage. This paper considers only the ECG values as it plays vital role in diagnosing carious cardiac diseases.

T. N. Gia et al. [25] proposed a system that handles the issues like high energy consumption using a fog network.
The concept of fog computing reduces the load of cloud computing by computing the data near to the edge devices and storing the data distributed storage and also reduce power consumption by $50 \%$ and also provides the features such as near to edge computing and distributed data storage.

Fog computing mainly helps to overcome the disadvantages of cloud computing. Similarly, there are some challenges in healthcare industry like converting big data into smart data, geographical distribution of providers and patient's privacy and security. Fog computing helps us to overcome this challenges in a simple way.

Below, in Table I there are some effective solution for the challenges of IoT [22]

\section{Table I.Solving The Challenges Of Iot}

\begin{tabular}{|l|l|}
\hline IoT Challenges & Solution using Fog \\
\hline Latency & $\begin{array}{l}\text { Fog performs the time } \\
\text { consumption tasks such as } \\
\text { analysis and control near to the } \\
\text { user. }\end{array}$ \\
\hline Network Bandwidth & $\begin{array}{l}\text { Fog will not send all the data to } \\
\text { the cloud. It restricts the data to } \\
\text { be sent, instead it will store the } \\
\text { data locally for quick access. }\end{array}$ \\
\hline Uninterrupted connectivity & $\begin{array}{l}\text { Fog provides an extra feature } \\
\text { where the fog nodes can } \\
\text { communicate even if there is no } \\
\text { intermittent network } \\
\text { connectivity to cloud. }\end{array}$ \\
\hline New IoT security challenges & $\begin{array}{l}\text { A fog system can act as proxy, } \\
\text { perform range of security } \\
\text { functions manage security } \\
\text { status, can detect threat using } \\
\text { local information. }\end{array}$ \\
\hline
\end{tabular}

The above solutions can also be stated as the advantages of fog computing.

\section{BIOMEDICAL SENSORS AND MICROCONTROLLERS SUITABLE FOR RECORDING BIO-SIGNALS}

In this part of paper discusses about different kinds of biomedical sensors that can be used to sense patient vital information and also discusses about the different kind of fog nodes or microcontrollers used to record and transmit the data.

\section{ECG Sensors}

\section{AD8232 Heart Monitoring Sensor}

AD8232 is a biomedical sensor which is used to sense the electrical activity of the heart. Electrical activity of the heart can be graphically represented in the form of ECG. AD8232 is a single lead sensor which consists of 3 removable electrode pads. The sensor sense the electrical signals of the heart and sends it to the microcontroller as an analog value. This analog value will be converted to digital using the inbuilt ADC (Analog to Digital Converter) on microcontroller.

The microcontrollers like Arduino and Raspberry Pi can be used to record the data. AD8232 can be directly connected to Arduino. If we use Raspberry pi we need an external ADC to be attached because the Raspberry Pi doesn't have inbuilt ADC. 


\section{Fog Based Sensing and Transmission Mechanisms of Vital Bio-Signals}

AD8232 has six pins,

- GND - Used to Ground the circuit.

- $3.3 \mathrm{v}-$ Takes $3.3 \mathrm{v}$ power supply.

- Output - Used to send sensed analog values.

- LO- - Lead-Off detect negative.

- LO+ - Lead-off detect Positive.

- $\mathrm{SDN}-\mathrm{This}$ is not often used. It is used to shut down the system.

Connection with Arduino,

- Sensor GND to Arduino GND.

- Sensor 3.3v to Arduino 3.3v.

- Sensor Output to Arduino A0.

- Sensor LO- to Arduino 10.

- Sensor LO+ to Arduino $\sim 11$.

\section{DFRO bot Heart rate monitoring Sensor}

This biomedical sensor is used to measure the electrical activity of the heart. This can be graphically charted as ECG and this biomedical sensor outputs the analog value. An ECG signal can be very noisy, to avoid that AD8232 chip has been added for the sensor to generate clear PR and QT intervals. This sensor can be used with the microcontrollers like Arduino and Raspberry Pi.

Connection with Arduino,

- Sensor 5v to Arduino 5v.

- Sensor GND to Arduino GND.

- Sensor Analog pin to Arduino Analog pin.

\section{PulseRate Sensors}

\section{Pulse Sensor}

Pulse sensor is a biomedical plug and play sensor used to sense the Pulse rate or Beats per minute. This sensor also adds amplification, noise reduction and gives an accurate pulse rate. Pulse Sensor consists of 3 pins i.e. Positive pin used for taking $3.3 \mathrm{v}$ power supply, negative pin used for Ground connection and S pin which is used to send output to the microcontroller. Pulse Rate sensor can be used with the microcontrollers like Arduino and Nodemcu.

\section{Connection with Arduino or Nodemcu,}

- Sensor positive pin to Arduino 3.3v.

- Sensor negative pin to Arduino GND.

- Sensor S pin to Arduino A0.

\section{Max30100 Pulse oximeter}

Max30100 Pulse oximeter is used to sense both pulse rate and oxygen saturation i.e. Spo2. This sensor takes a pulse rate once it recognizes the pulse beat and has IR led and red Light led which is used to get the saturation of the blood. There are 2 kinds of sensors, the first kind is normal Max30100 which need 4.7omh resistor to pull the resistance i2c pins on the module when connected with Arduino[26]. The other kind is GY-Max30100 which is built for the Arduino and this module doesn't require any resistor. This module or sensor can be used with the microcontrollers like Arduino and Raspberry Pi.

\section{Connection with Arduino,}

- Sensor GND to Arduino GND.

- Sensor SDA to Arduino A4 operates from 1.8 to 3.3v.

- Sensor SCL to Arduino A5 operates from 1.8 to 3.3v.

- Sensor vin to Arduino 5v.

\section{Muscle activity sensors}

\section{Myoware muscle activity sensor}

Myoware muscle sensor is one of the biomedical sensor which is cost effective and it helps to predict mayocardial infarction by mesuring the muscle activity. This sensor provides an output voltage between 0 to $5 \mathrm{v}$ considering the amount of activity in a muscle. This sensor can be connected to the microcontroller like arduino [27].

\section{Connection with Arduino,}

- Sensor GND to Arduino GND.

- Sensor +vs to Arduino 5v.

- Sensor output to Arduino a0.

\section{Muscle sensor v3}

This is a biomedical sensor used to measure the muscle activity. This sensor can be used with any microcontroller which has inbuilt analog to digital converter. The signals recorded by this sensor can be graphically represented as electromyography (EMG). Same as ad8232 heart monitoring sensor this also has 3 electrode pin which records the muscle activity.

Connecting with Arduino.

- $\quad+$ and -pin of sensor to $9 \mathrm{v}$ battery.

- Output pin of sensor to a0 of Arduino.

- GND of sensor to GND of Arduino.

\section{Microcontrollers}

\section{Arduino UNO Microcontroller}

Arduino UNO is microcontroller based of ATmega328. It is low cost and easily available microcontroller. This microcontroller has 13 digital pin and 6 analog pin and can connect external power source up to $12 \mathrm{v}$. It has $32 \mathrm{~kb}$ flash memory and $16 \mathrm{MHz}$ clock which is fast enough for most application. This microcontroller has $5 \mathrm{v}$ and $3.3 \mathrm{v}$ power supply pin. This Microcontroller does not have inbuilt BT (Bluetooth) or Wi-Fi module. We can add HC-05 to the board to enable BT. HC-05 is an external Bluetooth module which enables the BT option for a connected board. The data can be transmitted or received serially using RX and TX.

\section{Connecting HC-05 with Arduino,}

- HC-05 GND to Arduino GND.

- HC-05 5v to Arduino 5v.

- HC-05 RX to Arduino TX.

- $\mathrm{HC}-05$ TX to Arduino RX.

We can enable Arduino with Wi-Fi using Nodemcu or ESP866 Module. Nodemcu is kind of Microcontroller with inbuilt ESP8266 Wi-Fi module used to enable the Wi-Fi capability of the connected board.

\section{Connecting Nodemcu to Arduino,}

- Nodemcu GND to Arduino GND.

- Nodemcu TX to Arduino RX.

- Nodemcu RX to Arduino TX.

- Nodemcu can be powered using USB cable.

\section{Raspberry Pi}

Raspberry $\mathrm{Pi}$ is a credit card size microcontroller which has high processing capacity than the above mentioned microcontroller. Raspberry Pi is slower than 
the laptops and pc but has the capability to do the same task. Raspberry Pi is an open hardware which has the capability to run certain hardware like graphics, memory and USB. Raspberry Pi was built to run on Linux OS and also there are other 2 OS which can be used on Raspberry Pi they are, Raspbian and Pidora.

TABLE II.Difference Between Arduino And Raspberry

\begin{tabular}{|l|l|}
\multicolumn{2}{|c}{ Pi [28] } \\
\hline Arduino & Raspberry Pi \\
\hline $\begin{array}{l}\text { Arduino can only run one task } \\
\text { again and again. }\end{array}$ & $\begin{array}{l}\text { Raspberry Pi can run multiple } \\
\text { tasks. }\end{array}$ \\
\hline $\begin{array}{l}\text { Powering the Arduino using } \\
\text { battery pack is easy. }\end{array}$ & $\begin{array}{l}\text { Powering the Raspberry Pi using } \\
\text { battery pack is very difficult. }\end{array}$ \\
\hline Setup is easy. & $\begin{array}{l}\text { Setup is difficult should install } \\
\text { many libraries. }\end{array}$ \\
\hline Low cost microcontrollers. & It is expensive. \\
\hline $\begin{array}{l}\text { Arduino needs some external } \\
\text { modules to enable Wi-Fi options. }\end{array}$ & $\begin{array}{l}\text { Raspberry Pi has the inbuilt } \\
\text { Wi-Fi module and easy to } \\
\text { connect. }\end{array}$ \\
\hline $\begin{array}{l}\text { Arduino has onboard storage } \\
\text { capacity. }\end{array}$ & $\begin{array}{l}\text { Raspberry Pi does not have } \\
\text { onboard storage capacity but has } \\
\text { SD card slot. }\end{array}$ \\
\hline Arduino uses Arduino or C, C++. & $\begin{array}{l}\text { Raspberry Pi can be programmed } \\
\text { using Python, C, Ruby and etc. }\end{array}$ \\
\hline
\end{tabular}

Above, Table II depicts or compares both Arduino and Raspberry Pi microcontroller to understand which will suit the proposed system best based on cost and performance.

\section{PROPOSED SYSTEM}

The proposed idea is to model a secure, low cost and highly efficient system that makes the paramedic part automated in the ambulance and also record the vital data of the patient and traverse it to the hospital or to the doctor in time for decision making and also alert the doctor if the patient is critical during the time of travel (shift from hospital to another or home to hospital).

In Fig 4 the architecture can be viewed as 2 ends, one as the patient or ambulance end and the other as doctor end (mobile device). Ambulance end consists of the biomedical sensors to sense the patient vital data. The sensed data is then traversed to the fog node which also resides in the ambulance end. Here the data will be locally stored and then it will be traversed to the connected fog node or if there is an intermediary connection to cloud it will be directly stored in the cloud. Doctor end has an application which is capable of accessing the cloud to fetch data and display the same to the doctor.

For example, let us take a scenario where patient ' $\mathrm{A}$ ' is in the ambulance and if the system is not automated the paramedic should plug all the sensors to the patient and turn on each and every sensor to sense the data and this will take more time. But when the system is automated the only work of the paramedic should do is to plug the sensors to the patient body and click one button in the application, rest everything will be done by the system itself.

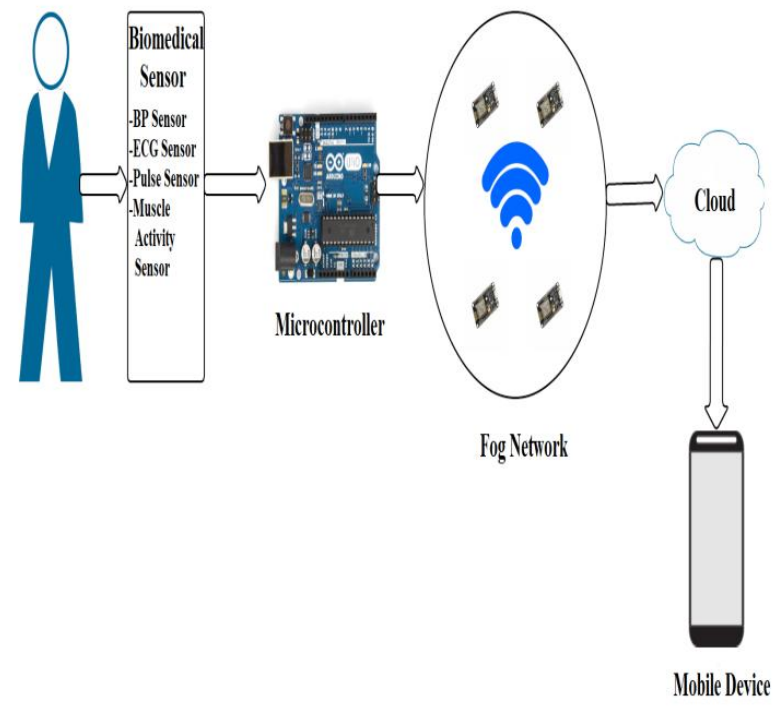

Fig 4. Basic Architecture of the Proposed Idea

Proposed System can be divided in to 4 parts Data Gathering, Data Transmission, Data Storage and Data Retrieval as shown in Fig 5.

\section{Data Gathering}

This part consists of different kinds of biomedical sensors. These sensors are connected to the microcontroller like Arduino to record the vital information of the patient. There are some challenges that we face while sensing the data and differentiate the sensed data - As all the sensors are integrated into a single board. All the sensors send the data at same time. To differentiate the data, we need to first set which sensor data should come first and which is next. It should also add the separator symbols like ',' or '|' after every sense.

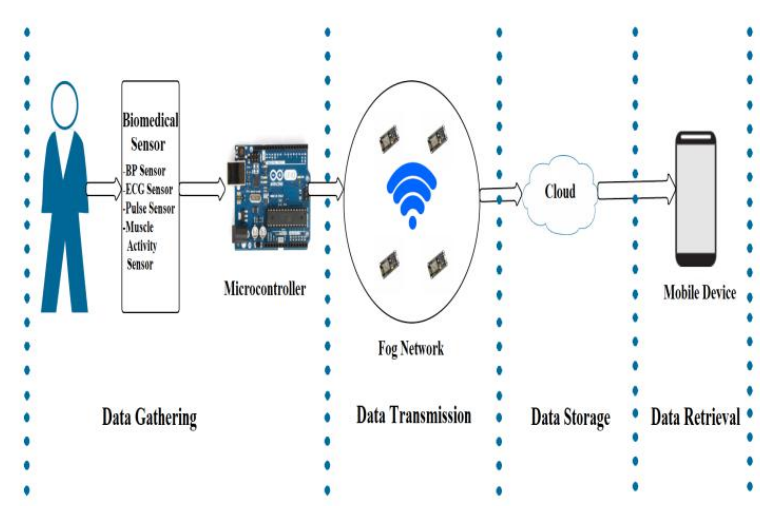

Fig 5. Architecture Breakdown

\section{Data Transmission}

Once the fog node gets the data it stores it locally and traverse it to the next nearest fog node or if there is an intermittent network to cloud the data will be stored in cloud. To find the nearest node we have referred Santosh Anand et al.[29],[30] paper which mainly focuses on finding the nearest neighbour node to traverse the data. Using this neighbour node the optimized roots to the destination can be found. Fog node acts both as the 


\section{Fog Based Sensing and Transmission Mechanisms of Vital Bio-Signals}

receiver and sender. If there is no network, communication will happen as shown below.

The communication happens in the following manner:

\section{Sender Side:}

Step1: Check for the data. If data exist act as a sender or else act as a Receiver.

Step2: Sense the nearest fog node using the access point name. If exists get connection using key.

Step3: Once the connection is established send the data. Step4: End connection.

\section{Receiver Side:}

Step1: Check for the data. If data does not exist act as a receiver or else act as a sender.

Step2: On the hotspot and act as an access point. Wait until the client is connected.

Step3: If client gets connected, read the data and store it in the memory.

Step4: End connection.

\section{Data Storage}

In the proposed idea the data storage happens in 2 locations.

- In the fog node for local processing and traverse.

- In the cloud for future use and for permanent storage.

The data stored in the fog node will be temporary because the fog node will have less storage capacity and acts just as a processing unit. Once its task is completed the fog node sends it to the nearest fog node or cloud and deletes the data from the memory. But in the cloud side the storage capacity will be high and can store the data permanently for future use.

\section{Data Retrieval}

Here comes the doctor's side or hospital side. The UI (User Interface) will be given to the doctor or the hospital person to view all the patients detail. The application in the doctor's side will fetch the data in real time and historical data present in the cloud. The application displays the data in an easily understandable way like using graph plots and with an attractive UI.

\section{Working Algorithm of Proposed System}

Input: Bio-signals recorded using different sensors.

Output: Storing recorded values in cloud and retrieving it.

//When paramedic clicks on Start Button.

Generate Session for patient;

Fetch Time;

While(Session)// if the session is on

\{

Fetch ECG;

Fetch Pulse Rate;

Fetch Muscle Activity;

Store record; \}

If (! Session) // if session is close. The session ends when paramedic clicks stop Button.

\{

Traverse (record);

\}

// Traverse method.

Traverse (record)
Search nearest Node;

Connect to nearest node;

Send (record);

The above algorithm tells how the proposed system sense the vital data and how the data is traversed to the healthcare centers quickly and accurately.

\section{CONCLUSION}

The examples given in the paper clearly states that technologies like IoT and Fog computing is solving many challenges of healthcare industry. There is a trend already going in healthcare industry to use IoT and there are many use cases emerging nowadays. The proposed system is also an example of such IoT projects where it avoids human intervention by automating the sensing part, minimize delay in transmission and allows user to access the data easily.

\section{ACKNOWLEDGMENT}

We feel deeply indebted to Her Holiness Most Revered Mata Amritanandamayi Devi (Amma) for her inspiration and guidance both in unseen and unconcealed ways. We also thank each and every staff at Amrita Vishwa Vidyapeetham, Mysuru campus for providing the necessary environment and encouragement for carrying out our work.

\section{REFERENCES}

1. "IOT Definition." [Online]. Available: https://www.happiestminds.com/Insights/internet-of-things/.

2. V. M. Rohokale, "A Cooperative Internet of Things ( IoT ) for Rural Healthcare Monitoring and Control," 2011.

3. A. A. Gurjar and N. A. Sarnaik, "Heart Attack Detection By Heartbeat Sensing using Internet Of Things : IoT,” pp. 3-6, 2018.

4. S. Monisha, R. Niranjana, M. Sabitha, and V. Sowmiya, "Iot based earlier stroke prediction," vol. 6, no. 03, pp. 118-122, 2019.

5. N. R. M, V. Prabu, R. Kumar, and M. Y. Ahamed, "IOT BASED EPILEPSY MONITORING AND DETECTION USING AMBULATORY SYSTEM," vol. 6, no. 03, pp. 2017-2019, 2019.

6. R. Harikrishnan, S. Keerthini, S. V Varshan, and R. Rahul, "IOT BASED SYSTEM DESIGN FOR DETECTING URINARY BLADDER LEVEL," vol. 6, no. 03,pp. 2017-2020, 2019.

7. B. N. Karthik, L. D. Parameswari, R. Harshini, and A. Akshaya, "Survey on IOT \& Arduino Based Patient Health Monitoring System," vol. 3, no. 1, pp. 1414-1417, 2018.

8. K. Yeh and S. Member, "A Secure IoT-Based Healthcare System With Body Sensor Networks," IEEE Access, vol. 4, pp. 10288-10299, 2017.

9. A. Mukhopadhyay, B. Xavier, S. Sreekumar, and M. Suraj, "Real-Time ECG Monitoring over Multi-Tiered Telemedicine Environment using Firebase," 2003.

10. D. N, R. K, R. Krishnan, and M. Ramesh, "A Low Cost Remote Cardiac Monitoring Framework for Rural Regions," Proc. 5th EAI Int. Conf. Wirel. Mob. Commun. Healthc. - "Transforming Healthc. through Innov. Mob. Wirel. Technol., 2015.

11. J. S. Kumar, "A Survey on Internet of Things : Security and Privacy Issues," vol. 90, no. 11, pp. 20-26, 2014.

12. C. Doukas and I. Maglogiannis, "Bringing IoT and cloud computing towards pervasive healthcare," Proc. - 6th Int. Conf Innov. Mob. Internet Serv. Ubiquitous Comput. IMIS 2012, pp. 922-926, 2012.

13. M. Hassanalieragh et al., "Health Monitoring and Management Using Internet-of-Things (IoT) Sensing with Cloud-Based Processing: Opportunities and Challenges," Proc. - 2015 IEEE Int. Conf. Serv. Comput. SCC 2015, pp. 285-292, 2015.

14. "IoT products." [Online]. Available: 
https://econsultancy.com/internet-of-things-healthcare/.

15. "Benefits of IOT in Health care." [Online]. Available: https://www.archer-soft.com/en/blog/5-benefits-internet-things-hosp itals-and-healthcare.

16. "Fog computing Definition." [Online]. Available: https://internetofthingsagenda.techtarget.com/definition/fog-computi ng-fogging

17. T. Patel and K. Jariwala, "Fog computing in iot 1 1," pp. 17-21, 2018.

18. J. Abdelaziz, M. Adda, and H. Mcheick, "An Architectural Model for Fog Computing," vol. 10, no. 1, pp. 21-25, 2018.

19. R. Mahmud, K. Ramamohanarao, and R. Buyya, "Latency-Aware Application Module Management for Fog Latency-aware Application Module Management for Fog Computing Environments," no. March, 2018

20. [20] G. Lee, W. Saad, and M. Bennis, "An Online Secretary Framework for Fog Network Formation with Minimal Latency,” pp. 1-6, 2017.

21. S. Sarkar, S. Member, S. Chatterjee, and S. Member, "Assessment of the Suitability of Fog Computing in the Context of Internet of Things," vol. 7161, no. c, pp. 1-14, 2015.

22. M. Chiang and T. Zhang, "Fog and IoT: An Overview of Research Opportunities," IEEE Internet Things J., vol. 3, no. 6, pp. 854-864, 2016

23. Y. N. Krishnan, C. N. Bhagwat, and A. P. Utpat, "Fog ComputingNetwork Based Cloud Computing," no. Icecs, pp. 250-251, 2015.

24. T. N. Gia, M. Jiang, A. M. Rahmani, T. Westerlund, P. Liljeberg, and H. Tenhunen, "Fog computing in healthcare Internet of Things: A case study on ECG feature extraction," Proc. - 15th IEEE Int. Conf. Comput. Inf. Technol. CIT 2015, 14th IEEE Int. Conf. Ubiquitous Comput. Commun. IUCC 2015, 13th IEEE Int. Conf. Dependable, Auton. Se, pp. 356-363, 2015.

25. T. N. Gia et al., "Fog Computing in Body Sensor Networks: An Energy Efficient Approach," IEEE Int. Body Sens. Networks Conf. no. August, 2015

26. "MAX30100." [Online]. Available: https://www.instructables.com/id/How-to-Wire-MAX30100-Heart-R ate-Monitor-With-Ardui/.

27. "Myoware muscle sensor." [Online]. Available: https://randomnerdtutorials.com/9-biometric-sensors-arduino-compa tible/.

28. "Difference between Arduino and Raspberry pi."

29. S. Anand and A. Rr, "A Protocol for The Effective Utilization of Energy in Wireless Sensor Network," vol. 7, pp. 93-98, 2018.

30. S. Anand and P. A. Radhakrishna, "A Protocol for the Effective Utilization of Energy in Wireless Sensor Network," vol. 7, pp. 82-86, 2018 . 